\title{
ERRATUM
}

\section{Propagation and Dissemination of Infection after Vaginal Transmission of Simian Immunodeficiency Virus}

Christopher J. Miller, ${ }^{1,2,3,4 *}$ Qingsheng Li, ${ }^{5}$ Kristina Abel, ${ }^{1,2}$ Eun-Young Kim, ${ }^{6}$ Zhong-Min Ma, ${ }^{1,2}$ Stephen Wietgrefe, ${ }^{5}$ Lisa La Franco-Scheuch, ${ }^{1,2}$ Lara Compton, ${ }^{1,2}$ Lijie Duan, ${ }^{5}$ Marta Dykhuizen Shore, ${ }^{5}$ Mary Zupancic, ${ }^{5}$ Marc Busch,, ${ }^{1,2}$ John Carlis, ${ }^{5}$ Steven Wolinsky, ${ }^{6}$ and Ashley T. Haase ${ }^{5 *}$

Center for Comparative Medicine, ${ }^{1}$ California National Primate Research Center, ${ }^{2}$ and Department of Pathology,

Microbiology and Immunology, School of Veterinary Medicine, ${ }^{3}$ and Department of Infectious Disease, School of Medicine, ${ }^{4}$ University of California-Davis, Davis, California 95616; Department of Microbiology, University of Minnesota Medical School, Minneapolis, Minnesota 55455'; and Division of Infectious Diseases, The Feinberg School of Medicine at Northwestern University, Chicago, Illinois $60611^{6}$

Volume 79, no. 14, p. 9217-9227, 2005. Page 9217: The byline should appear as shown above. 